\title{
From describing to designing mathematical activity: the next step in developing a social approach to research in mathematics education?
}

\author{
Celia Hoyles \\ Mathematical Sciences Group \\ Institute of Education \\ University of London
}

Commentary on the Special Issue of Educational Studies in Mathematics 'Bridging the Individual and the Social: Discursive Approaches to Research in Mathematics Education'.

I am delighted and honoured to have been given the opportunity to provide a commentary on the papers presented in this special issue of Educational Studies in Mathematics, edited by Carolyn Kieran, Ellice Forman and Anna Sfard. It has given me the impetus to read with care accounts of research studies that define themselves as within the socio-cultural paradigm. ${ }^{1}$ The editors should be congratulated on bringing together a rich mix of papers that take different, but complementary, perspectives on the theme of the issue and together make a serious elaboration of the principles underlying this paradigm.

My starting point was as a learner. The papers collectively provided me with excellent summaries of a range of general theories underpinning the emerging social paradigm. I asked myself the following questions. What would the theoretical framing and methodologies of a socio-cultural approach add to the collective understandings developed in our field over the past thirty years? How can socio-cultural theory help us to understand and support students developing mathematical learning? Could I propose a novel slant on some of the ideas or analyses in the papers that might offer alternative but, to me at least, fruitful interpretative frameworks? Could I identify any omissions in analytic focus that, if addressed, might usefully form part of a future research agenda?

1 In the interests of clarity I have chosen to use the term socio-cultural throughout this commentary while recognising that others, including Cole, Wertsch, van Oers and Vygotsky, may use different terms. 
Given restrictions in space, my commentary cannot be exhaustive nor do justice to the wealth of insights offered in this rather large corpus of work. I have chosen therefore not to engage in theoretical discussion to reconcile (or not), for example, Vygotskian or Piagetian theories (some excellent discussions appear elsewhere, see Steffe and Thompson, 2000, Lerman, 1996, Cobb, 1996). Rather I choose to discuss theoretical issues only in so far as they have illuminated an agenda of a mathematics education researcher or, given the aim of this issue, served to move the community beyond the unproductive split between individual and social research perspectives.

In seeking to specify my initial goals, I must state the obvious. My commentary will be personal, inevitably shaped by my past experience and my research in mathematics education. So let me start with a personal comment. Nobody, least of all myself, would wish to deny the influence of the social perspective on mathematics teaching and learning. It is almost a truism to argue that all learning is shaped by history, power relations and culture, and that social forces transform classrooms and the way individuals interrelate and react in them. It is important to investigate both distally and proximally social phenomena (using categories distinguished by diSessa, personal communication), but equally important to distinguish between them. How far is it legitimate to restrict attention to one category of phenomena when researching mathematics education? Is it possible to embrace both categories in any investigation in anything but a superficial way?

Before turning to the papers in the volume, I briefly discuss my own professional career in the spirit of the socio-cultural paradigm, in order to inform the reader of the background to my remarks. ${ }^{2}$ In our book (Noss and Hoyles, 1996), Richard Noss and I commented that the community of mathematics education was little more than 25 years old, (now 30 years) but already, in this short time, there had been swings of methodologies, realignments of theoretical frameworks, and occasional paradigm shifts. We traced some of this history and noted a fundamental shift from a

2 Much of my work has been conducted collaboratively, most notably with Richard Noss whose contribution to all these ideas I acknowledge from the outset. 
focus on mathematical objects and how they were understood in the school population, initially, to a concern with strategies adopted during problem solving, later to a consideration of the construction of knowledge, and eventually to an acknowledgement of the essential complementarily in activity between process and content and of the importance of analysing the totality of mathematical experience. We noted how research had shown that taking the problem situation as the arbiter of meaning was fraught with pitfalls, not least because the mapping between the mathematical and situational elements of a problem turned out to be highly ambiguous, with respect to the mathematics deemed to be relevant, the aspects of the setting considered, and the extreme sensitivity of problem-meanings to social and cultural influences.

A key insight for our theoretical work at that time, was drawn from the seminal research of Vernaud (1982), Nunes, Carraher and Schliemann (1993) and Lave (1988), who had shown how mathematical meanings constructed within a setting were inextricably interwoven with their representations. Thus structure, context (meant more as physical rather than social setting at that time) and representation all comprised major pillars in our developing framework for understanding mathematics learning.

The next stage in my own research trajectory was a move from this largely cognitivist approach (tinged with concern about issues such as gender, and acknowledging the influence of teacher intervention), to one that included a socio-cultural perspective, in particular in investigations of the role of peer interaction with computer tools in learning mathematics. (Hoyles, Healy and Sutherland, 1991). From these studies, I argued that activity within specially-designed microworlds, ${ }^{3}$ shaped the interactions in the microworld, the tools of the microworld and the mathematical meaning's developed within and from these interactions. Representations and the tools or communicative devices with which they are intimately bound, could no longer be regarded as neutral players in the process of making meaning, a position consistent with the notion of 3 For a description of a microworld, see Hoyles (1993) and Edwards (1995). 
mediated action as elaborated by socio-cultural researchers, such as Wertsch, $(1991,1997)$ and Cole, (1996). But there were two dimensions central to this analysis that set it a little apart from these theorists: first, the activity in the microworlds was designed to foster mathematical meanings through construction, interaction and feedback, and second, the students could scaffold their own thinking ${ }^{4}$, through communicating with the tools of the microworld and shaping them, through programming, to fit their own purposes.

Through careful design of tools and of the interactions planned to take place in activities around these tools, we noted how students together constructed and reconstructed emergent ideas, and how we, as observers of their actions and their interactions in the form of written programs, gestures and verbal communications, were able to catch sight of this construction process as it took shape - this thinking-in-change. Thus, my research agenda focussed on the design of tools and activities for learning mathematics and how these worked out in practice, by reference to the ideas expressed by small groups of children. My goal was to investigate the transformative potential of tools and the co-evolution of tools and knowledge. One outcome of this research was the elaboration of the notion of situated abstraction, coined by Noss and myself, (Noss and Hoyles, 1992), as an attempt to capture how knowledge and symbolic technologies mutually constituted each other dialectically, through collective construction and negotiation.

While recognising the importance of the teacher in drawing attention to patterns of actions or symbols, or interesting variants and invariants in feedback, much of the research in computer-based settings could be described as cognitivist; concerned with students expressing their mathematics with the tools available. Mathematics, design and student interaction were the focal points of analysis ${ }^{5}$. Certainly my own work placed rather little emphasis on the wider classroom as a

4 Later Richard Noss and I further developed this idea of scaffolding under user control, in our notion of webbing, see Noss and Hoyles, 1996.

5 Research papers in this paradigm can be read in the International Journal of Computers for Mathematical Learning. 
community of practice, where norms are negotiated and understandings taken-as-shared (see for example Yackel \& Cobb, 1996), or where tools become integrated into ongoing mathematical work (see for example, Guin \& Trouche, 1999).

It is notable that these analyses of tool mediation in constructionist computer-based settings have tended to be separated from the recently popular socio-cultural trend in mathematics education, and in particular that of 'discursive psychology' (Harré \& Gillett, 1994): an example of the general tendency to isolate 'computer research' in a separate category from other research. Given my prior research and the limitations to which I have alluded, I am keen to ponder how bringing a discursive perspective to my research would allow a richer analysis; one which could take account of the influence of normative goals in the classroom, their interaction with students responses and developing ideas and their orchestration by the teacher, while not sacrificing the integrity of mathematical design.

So let me turn to the contributions in this special issue. In his paper, Lerman argues that the move to a cultural, discursive psychology enables the link between the actions of individuals and groups in the classroom and history and culture, and that such a move is necessary for educational studies (my emphasis). A strong claim, and one for which Lerman provides some theoretical justification. But how will this link be theorised in empirical studies in mathematics education is less clear. In contrast, Sfard takes mathematical discourse as her starting point and argues that one of the factors that makes this discourse special is 'its exceptional reliance on symbolic artefacts as its communication-mediating tools'. This aspect forms a central part of her case for regarding communication, not simply as an aid to thinking, but tantamount to thinking itself. Sfard insists that the metaphor of thinking-as-communication is a way of achieving a complementarity between the cognitive research tradition based on the metaphor of learning as acquisition, and the social-cultural framework around learning as participation. In this endeavour she appears to be at odds with 
Lerman. Sfard does not reject the idea of a cognitive invariant. Rather she moves on from an argument about the ontological nature of learning to a presentation of 'differing visions of the mechanisms of learning', visions emanating from individual or social analyses. In her detailed analysis of short extracts of student interaction, Sfard makes visible the competing influences on a child's response to mathematics: for example, his or her view of self in relation to mathematics, or the didactic contract with the teacher. Her dual analysis shows convincingly how any interpretative framework inevitably pre-judges 'findings', but, if different interpretative frameworks are used to compare and contrast and hone an argument, a researcher is better able to piece together the complex trajectory of thinking-in-change. Whose contributions are valued (or not) and why are just as important in the trajectory of learning as mathematically correct responses.

In reading Sfard's interpretations of the transcripts, I was struck by their plausibility - although I must admit occasionally to feeling that they tended to be over-judgmental (one boy was 'ignorant of this' or it was 'not making sense to him' ). I also wanted to add to Sfard's important reinterpretation of cognitive conflict as inter- or intra-discursive contradiction, a reference to the need for the prior establishment of a meta-rule for this conflict to be experienced; namely that statements in mathematics should be consistent and compatible.

Sfard not only presents her theory and illustrates its principle 'in operation', but also she describes in detail the new tools of analysis she has developed that have helped her to come up with her interpretations of the observed phenomena, tools that relate specifically to an analysis of the objectand meta-level aspects of discourse that she distinguishes. These methodological tools are in fact used to excellent effect in the contribution of Kieran that I shall discuss later.

But let me turn to another article, that by van Oers, who also makes explicit what he means by mathematical discourse, and makes a sustained effort to re-contextualise socio-cultural theories to 
study mathematical learning. Following Steinbring (1998) in describing 'mathematics' as a 'socially conventionalised discursive frame of understanding', van Oers acknowledges that 'not only factual technical mathematical operations are involved in mathematical activities in classroom, but epistemological constraints and social conventions are also part of the process'; and later, that 'doing and learning math means improving one's abilities to participate in mathematical practice, both the operational part (the symbolic technology of mathematics) and the discursive part'. In mathematics classrooms, utterances, for van Oers in a similar way to Sfard, are valued according to meta-rules and norms, as well as their literal meanings (a point van Oers acknowledges is not new and discussed by for example Cobb and his colleagues in many papers).

Analyses of the discursive rules that regulate communication in mathematics classrooms, and which draw attention to the teacher who introduces and monitors these rules, appear as a central strand in socio-cultural research. Sfard's paper adds a further dimension, since she attempts to bring together analysis of content with that of communication. Not only does she describe, like van Oers, 'the meta-discursive rules that regulate the communicative effort', but also twins this analysis with a consideration of 'the mediating tools (or simply mediators) that people use as a means of communication'. She argues that 'tools are shapers of content, that is, of the object-level aspects of discourse and meta-discursive rules are the moulders, enablers and navigators of the communicational activities.' It appears that object-level aspects are the bridges to a more cognitivist-oriented and individual approach, which could stand alongside and complement the social analysis, while preserving the discursive nature of both.

Returning to analyses of the regulation of interactions in classrooms, Bakhtin's notion of speech genre is used, to good effect to see, in van Oers' terms, how 'people's utterances in a communication process are not only regulated by the processes that occur in the direct interaction, but also by the historically developed style of communicating in the particular community of practice' (his 
emphasis). It is through interaction with a teacher, often revoicing ${ }^{6}$ 'relevant' contributions that, van Oers argues, students come to interiorise the rules that regulate the discourse of mathematics - to be systematic, consistent, symbolic, abstract. Revoicing is a distinctive methodological tool in the socio-cultural paradigm: a teacher will 'repeat, expand, recast, or translate student explanations for the speaker and the rest of the class' (Forman and Ansell, this issue), and it is in this process that she/he defines what is preferred and allowable.

Abstractness, van Oers suggests, is the hallmark of mathematical thinking. Perhaps he is right, but maybe this is an idea that sits rather problematically alongside a socio-cultural approach and is certainly a term that is hotly debated (see for example Schwartz, 2001). In the Vygotskian School for example, emphasis is placed on connections between signs, and mathematics appears as the epitome of decontextualisation, the pinnacle of abstraction. Bakhtin/Volosinov suggests that: 'What interests the mathematically-minded rationalists is not the relationship of the sign to the actual reality it reflects nor the individual who is its originator, but the relationship of sign to sign within a closed system already accepted and authorised. In other words, they are interested only in the inner logic of the system of signs itself, taken, as algebra, completely independent of the ideological meanings that give the signs their content.' (Volosinov, 1973, pp. 57-58).

Thus, to me, the Vygotskian tradition appears to point to mathematical discourse as a unique form, contrasting with all other sign systems. It draws attention to the ways in which meaning is produced in terms of intra-mathematical relations, in sign-sign mediation, and suggests that this is the only mechanism for the production of mathematical meaning: there is no effective role either for other symbol systems, or for interaction with social or physical reality (see also Confrey, 1995). If this were the case, we might at least go some way to explaining the difficulty with which so many are enculturated into mathematical discourse, but we would do so by erecting (or maintaining) a rigid barrier between social and practical activity on the one hand, and mathematical thought on the

6 A term coined by O'Connor and Michaels, 1996. 
other. So a concern that permeated all my reading was about the place in this paradigm of new (or alternative) mathematical epistemologies, possibly brought into being by the presence of new tools. How are new meta-rules and norms and new operational procedures introduced and researched? I will return to this point later, but for the moment, trace in other papers analyses of how the culture of a mathematics classroom is developed and how the teacher enculturates students into what is allowed as mathematical and what is not.

As well as presenting a theoretical framework based on cultural psychology, Forman and Ansell $\backslash$ rquote s contribution defines a methodology emanating from this framework, explicitly and in detail. It involves distinguishing episodes in classroom interaction and times of transition, along with care to establish the generalisability of any single case analysed. Their research brings the personality and personal history of the teacher into the analysis of classroom interaction as another tool in the interpretation of her regulation of the classroom dialogue. Forman and Ansell again use the notion of revoicing most productively to recognise changes in the structure of a teacher's discourse, changes that may well have remained hidden in studies within another paradigm. By analysis of the discourse in a classroom community and by placing the individual teacher in her social context, the authors are able to distinguish two distinct voices: one that occurred during discussions of students' invented strategies and the other that emerged during talk about standard algorithms.

Turning to another classroom study, O'Connor set out to understand how the web of mathematical content at the focus of a position-driven discussion ${ }^{7}$ might interact with its linguistic formulations, and the constraints and affordances of activity structures. The question under discussion was, 'can any fraction be turned into a decimal'? What I took from this text is a picture of an expert teacher orchestrating discussion around this mathematical question, with all its potential meanings; she

7 A discussion involving a teacher leading a group of students in exploring a central question with a limited number of answers. 
generated mathematical dialogue - by encouraging students to find and test counter-examples and by introducing strategic examples to open up new questions or lines of enquiry - and, at the same time, she built a mathematical community - by distinguishing personal disagreement from mathematical disagreement, monitoring what was 'taken-as-shared', and revoicing confusion. What was new to me too was the explicit discussion of the times when the teacher 'mis-interpreted' a student remark or was unable to make sense of it, and the repertoire of face-saving moves in the discourse that she might use. I did however miss any individual perspective: for example (following Sfard's analysis), I wondered if the role of counter-example was actually appreciated by the students?

O'Connor' s study, like Forman and Ansell $\backslash$ rquote s, reveals a phenomenon that might well have remained hidden without her analytic tools: that the teacher's strategies varied, not this time in response to different student contributions, but according to phase of lesson. At times of review where ideas were widely shared, the teacher reorientated her interactions to focus on the precision and accuracy of language as a central part of the discourse of mathematics.

In contrast, during exploratory discussion, criteria to evaluate student responses were deliberately loose, so students could 'solidify their knowledge and practice their ability to verbally articulate what they know' . I pondered this interpretation and how it fitted with principles of socio-cultural research, since it gave me the impression of 'knowledge in one's head'.

Following this thought and pursuing a more individual line of enquiry in relation to tool mediation alongside the social, I would have liked to see more analysis in O'Connor's study of the use of the calculator, and how this use might have mediated the meanings the students developed, alongside the dialogue with the teacher. For example, how did the physical limitations of the size of the calculator's window shape students' responses, and what was the status of one student's conjecture 
apparently derived from the availability of the buttons on a calculator that would allow him to convert any fraction to a decimal? I raise this point here, not to insist that the author should have followed up this analysis, but rather to show that while choices must be made, they can (as in this case) leave open avenues for future exploration.

In a third classroom study, again researched against a background of Vygotskian and Bakhtinian social psychology, Zack and Graves add yet another dimension to socio-cultural analysis, namely that of teacher as learner. Again we read of how an expert teacher builds a community where students are expected to conjecture, listen to each other, argue and justify their reasoning in ways that acknowledge others' contributions. The main body of the paper is an analysis of three boys engaging with two open-ended problems against a backdrop of their work in previous problems. In effect, the teacher in setting the problems was seeking to provoke the group to recognise the structural (mathematical) equivalence of 'the diagonals problem' and 'the tunnels problem', an equivalence already noticed by one of the boys. The paper describes the boys' use of mathematical language and concepts, and their evolving understanding, through discussion and argument, of an algebraic expression constructed by one of the children. The analysis focuses on the different roles the boys take, for example, to seek generalisations and encode in algebra, or to seek explanations. It also draws attention to the fact that the teacher understood the analogical relationship conjectured by one boy in a rather different way than was in fact the case, a mismatch that may have led to the impasse described but also led the teacher 'to learn'.

But could the analysis be interpreted in a different way and how could it throw light on what is for me a fascinating question; the question of 'transfer' approached from a socio-cultural perspective? A more cognitivist approach alongside the socio-cultural might have focussed on the use of algebra as a means of expression and of communication (or mis-communication) between the students. Algebra appeared to be the 'expected way' to encode the relationships perceived (a meta-discursive 
rule?), rather than a language for students to discuss, negotiate and manipulate. I would interpret what an individual boy had constructed interactively and externalised as the algebraic description of the number of diagonals or the number of tunnels as a situated abstraction: an interrelated product of constructed knowledge and algebraic expression. Written algebra framed and constrained what the boys 'saw', but at the same time, might also have served as a catalyst for 'seeing the connections', if a discursive move (by the teacher) had been made to shift attention from signreferent connections to sign-sign connections.

Zack and Grave's research prompted me to return to the work of Balacheff (1991), who several years ago analysed (slightly older) students working on a similar diagonals problem. I wanted to compare his, constructivist and Lakatosian perspective with the socio-cultural approach. The comparison and contrasts turned out to be too numerous for me to elaborate here - it was a fascinating experience. But I simply mention a few differences: differences in research context, that is experimental and 'everyday' classroom; differences in what is produced as evidence; differences in how far the children and the teacher are given personal voices; and differences in interpretation of 'the acceptance' of a counter-example (again Sfard's analysis is a useful reference). Making the comparison also highlighted how hard it was for me to trace how the meta-theoretical tools used in Zack and Grave \rquote s study, namely those of 'semantic discourse analysis, sociolinguistic and conversational analysis and models of informal reasoning' were actually operationalised in practice. This is a problem that must be faced by all researchers adopting this paradigm, since inevitably only illustrative data can be presented in any one article.

Methodological approach and analysis is indeed visible in the contribution of Kieran in her analysis of the mathematical discourse of 13-year-old partners solving a mathematical problem. The work consisted of joint problem-solving, followed by individual report writing and then individual work on problems analogous to those worked on jointly. My interest in this paper was more than as a 
commentator, as I have been involved in rather similar research with group and individual work of similar-aged children, although in my studies the computer was always used for joint problem solving, while in Kieran's research its use was optional. (See for example Hoyles, Healy and Pozzi, 1994; Healy, Pozzi and Hoyles, 1995).

Kieran used what she called an interactivity flow chart, 'to synthesise from the transcripts the ways in which students interacted with each other, and to permit the researcher not only to detect at a glance the nature of the interactions but also to focus attention on those utterances that seemed to develop the mathematical content of the discourse' (my emphasis). The analysis (following Sfard) distinguished between different channels of communication (personal and interpersonal) and different levels of talk (object-level and non-object-level). Thus Kieran focuses on a major dilemma of linking public and private discourse, by looking in detail at the interactions of children around a challenging task. The transcripts made it possible to trace how knowledge was collectively constructed and to conjecture reasons for discrepancies between partners in their subsequent individual responses - an analysis I had not seen before. Kieran' s conclusions are worthy of further research, namely that; 'The patterns of interaction that were found to be most productive for both members of the pairs were those where the interpersonal channel was the site of frequent objectlevel utterances. Those interactions where it was the personal channel of only one of the participants that was the main site of the publicly-uttered thinking--utterances that were neither complete nor ever expanded upon--were not conducive to the emergence of mathematics for both participants'.

This paper, as with many others, left me pondering about where it sat in the socio-cultural paradigm, and the fruitful lines of research it opened up. For example, in the follow-up individual work, how were problems deemed to be 'analogous' from this perspective to those the students worked on with a partner? Also, what were the meta-rules regulating the student work, in terms of 
what was valued, that is their joint products or their individual work. ${ }^{8}$ What was the influence of a school culture where questions are presented in logical sequence, thus enabling 'copying' ? What was the role of the computer in mediating the interchanges of the students? I will elaborate on this last issue. From my reading of the paper, computer use seemed to prompt, not only a change in patterns of interaction between one of the pairs, but also a change in style of problem solving, to one involving trial and evaluation, where the trial externalised the thinking of one of the pair in a public way for the other boy to build upon. Kieran, in fact, drew attention to computer mediation: 'and it was at this time [when the pair was actively involved with graphic software] that the interpersonal channel became alive with object-level utterances'. A complementary, individual approach might take this mediation as central and as the interpretative frame of the interactions.

So how can I summarise my reactions to the volume? The socio-cultural paradigm as represented in research reported in these papers is beginning to clarify what this theory can offer mathematics education. I was relieved to find that most authors did not seek to erase the individual perspective, and by their focus on communities of practice did not necessarily deny the integrity of an individual's reasoning. As Sfard argued: 'rather than rejecting the long-standing acquisition metaphor, we should supplement it with theories grounded in alternative metaphors'. Focussing on any one effort will inevitably limit analysis of others; this is the case if we simply look at the social side, as much as if we simply look at the individual. I see no argument for prioritising one over the other. However, I do insist that studies in mathematics education should involve some discussion of mathematical activity, however this is defined. There are invariances to our discipline that we cannot, and should not, ignore.

If we take the zooming metaphor seriously, as Lerman suggests, we must allow the researcher to zoom to interactions of individuals during mathematical activity, and while recognising its

8 The distinction between working 'for the group' or for one's own individual learning was found to be crucial in my research referred to earlier. 
limitations not necessarily analysing them in the same study. I do not believe it possible or even desirable that 'the goals and desires that are associated with the multiple practices of the classroom must form part of the analyses we carry out' (Lerman, 1996, my emphasis).

To mention social issues in largely cognitive work, all too easily leads merely to descriptive padding, not used in subsequent analysis. Despite my disagreement with Lerman on this point, he does mention an important set of potential influences on mathematics learning, each of which could usefully be the subject of research or linked to research in other paradigms: for example, class and gender (both notably absent in empirical analyses in this volume), and also tool mediation. Referring to the work of Bartolini-Bussi in relation to the drag mode in dynamic geometry, Lerman mentions that 'internalising the tool transforms the way one can act enabling conjectures to be generated, for example that are unique to the dynamic geometry environment, as a result of the tool' . Research in the constructionist paradigm that I have mentioned earlier has in fact explored this idea in depth. For example, the complexities of the drag mode in use have been extensively analysed by, for example, Hölzl, 2001: the tool is not one object but is constructed differently by the learner community in different activities. If work in these different paradigms on tool mediation could build upon each other, this would be a huge step forward for our community.

Discussion of tool mediation as a unit of analysis was largely missing in this corpus of work, a remark largely referring to mediation by computer tools, but not necessarily limited to these: also absent from these analyses was reference to almost any means of interaction other than the verbal, written communication to take an example. It may be that establishing and elaborating a toolmediation focus would help to build bridges between the individual and the social. This takes me back to a point I made earlier about new developments. Much of the research presented in this issue analysed and interpreted what was taking place in activities in classrooms. What I missed was any discussion of the design of the activities and the design or choice of the tools or sign systems that 
were introduced to foster mathematics learning. It is not, of course, that design will lead to outcomes in a deterministic way, but at least this focus would allow investigation of the transformative potential of tools in activities (see diSessa, Noss \& Hoyles, 1995, and more recently, Cobb, 2000). Teachers not only shape the culture in the classroom, but also (with researchers) can play an active role in changing this culture- through organising the tasks and activities at an object level, as well as through interactions at a meta-discursive level. Most crucially, acknowledging design brings knowledge and epistemology back into centre stage. 


\section{References}

Balacheff, N., (1991). Treatment of Refutation: Aspects of the Complexity of a Constructivist Approach to Mathematics Learning, in Glasersfeld, E., (ed), Radical Constructivism in Mathematics Education, 89-110, von Netherlands: Kluwer.

Cobb, P., Wood, T., and Yackel, E., (1993). Discourse, mathematical thinking and classroom practice, in Forman, E.A, Minick, N. and Stone, C.A., (eds.), Contexts for learning. Sociocultural dynamics in children's development, 91-119, New York: Oxford University Press.

Cobb, P., (1996) Constructivism and activity theory: A consideration of their similarities and differences as they relate to mathematics education. In Mansfield, H., Pateman, N., \& Bednarz N., (eds), Mathematics for Tomorrow's Young Children: International perspectives on curriculum , 1056. Dordrecht, Netherlands: Kluwer.

Cobb, P., (2000). The Importance of a Situated View of Learning to the Design of Research and Instruction, in Boaler, J., (ed) Multiple Perspectives on Mathematics Teaching and Learning , 4582, Ablex Publishing, Westport, CT, USA.

Cole, M., (1996). Cultural Psychology: A Once and Future Discipline, Cambridge MA.: Belknap Press of Harvard University Press.

Confrey, J., (1995) Theory of Intellectual Development, Part 3, in For the Learning of Mathematics, 15, 2 (June), 36-48.

diSessa, A., Noss, R., and Hoyles, C., (1995) (eds.) Computers and Exploratory Learning, Berlin: Springer-Verlag.

Edwards, L.D., (1995). Microworlds as Representations, in diSessa, A., Noss, R., and Hoyles, C., (eds.) Computers and Exploratory Learning, 127-194, Berlin: Springer-Verlag.

Guin, D., \& Trouche, L., (1999). The complex process of converting tools into mathematical instruments: the case of calculators. International Journal of Computers for Mathematical Learning, 3, 3, 195-227.

Harré , R., and Gillet, G., (1994). The discursive mind. London: Sage.

Healy, L., Pozzi, S., and Hoyles, C., (1995). Making sense of groups, computers and mathematics. Cognition and Instruction, 13, 4, 505-523.

Hölzl, R., (2001). Using dynamic geometry software to add contrast to geometric situations: A case study. International Journal of Computers for Mathematical Learning, 6, 1, 63-86.

Hoyles, C., Healy, L., and Sutherland, R., (1991). Patterns of discussion between pupil pairs in computer and non-computer environments. Journal of Computer Assisted Learning, 7, 210-228.

Hoyles, C., (1993) Microworlds/schoolworlds: the transformation of an innovation, in Keitel, C., and Ruthven, K., (eds.) \}Learning from Computers: Mathematics Education and Technology, NATO ASI Series, 1-17. Berlin:Springer-Verlag.

Hoyles, C., Healy, L., and Pozzi, S., (1994). Groupwork with computers: an overview of findings. Journal of Computer Assisted Learning, 10, 202-215. 
Hoyles, C., and Noss R., (1996). Windows on Mathematical Meanings: Learning Cultures and Computers, Netherlands: Kluwer.

Lerman, S., (1996). Articulating theories of mathematical learning: A challenge to the radical constructivist paradigm? Journal for Research in Mathematics Education, 27, 2, 133-150.

Lave, J., (1988). Cognition in Practice. Cambridge: Cambridge University Press.

Noss, R., and Hoyles, C., (1992). Looking back and looking forward, in Hoyles, C. and Noss, R. (eds.) Learning Mathematics and Logo,431-46, Massachusetts: Massachusetts Institute of Technology.

Nunes, T., Schliemann, A.D., and Carraher, D.W., (1993). Street Mathematics and School Mathematics. Cambridge: Cambridge University Press.

O'Connor, M.C., \& Michaels, S., (1996). Shifting participants frameworks: orchestrating thinking practices in group discussion. In Hicks, D., (ed), Discourse, learning and schooling, 63-104. Cambridge: Cambridge University Press.

Steffe, L. P., and Thompson, P. W., (2000). Interaction or Intersubjectivity? A reply to Lerman. Journal for Research in Mathematics Education, 31, 2, 191-209.

Schwartz, B., (ed), (2001) Abstraction and Context, Special Issue of Quarterly of Cognitive Science, 1, 3 \& 4, 237-424,

Steinbring, H., (1998). Mathematical understanding in classroom interaction: the interrelation of social and epistemological constraints, in Seeger, F., Voight, J., \& Waschescio, U., (eds), The culture of the mathematics classroom, 344-372, Cambridge: Cambridge University Press

Vergnaud, G., (1982). Cognitive and developmental psychology and research in mathematical education: some theoretical and methodological issues. For the Learning of Mathematics,3, 23, 3141.

Wertsch, J.V., (1991). Voices of the Mind: A Sociocultural Approach to Mediated Action. Harvard University Press, Cambridge, MA.

Wertsch, J.V., (1997). Mind as Action, New York: Oxford University Press.

Yackel, E., and Cobb, P., (1996). Sociomathematical norms, argumentation, and autonomy in mathematics, Journal for Research in Mathematics Education , 27, 458-477. 